(Appl. Phys. Lett. Vol.62, No.18, 2155-2157 (1993))

\title{
Whispering-gallery-mode dye lasers in blue, green, and orange regions using dye-doped, solid, small spheres
}

\author{
Hiroshi Taniguchi, Tamiya Fujiwara, and Hiroshi Yamada \\ Department of Electrical Engineering, Faculty of Engineering. Iwate University, 4-3-5 Ueda, \\ Morioka-shi 020, Japan \\ Shinji Tanosaki and Mamoru Baba \\ Department of Electronic Engineering, Faculty of Engineering, Iwate University, 4-3-5 Ueda, \\ Morioka-shi 020, Japan
}

(Received 9 September 1992; accepted for publication 1 March 1993)

\begin{abstract}
A whispering-gallery-mode (WGM) dye laser in blue, green, and orange regions is reported using dye-doped, solid, small spheres. A WGM dye laser is pumped by a transversely excited atmospheric UV $\mathrm{N}_{2}$ laser. Some features of the WGM dye laser are demonstrated.
\end{abstract}

A dielectric sphere possesses natural modes of oscillation at characteristic frequencies corresponding to specific size-to-wavelength ratios defined as a size parameter. $\mathrm{Re}$ cently, the advantages of the spherical resonator have been pointed out because of the very high-quality factor ( $Q$ value) of electromagnetic whispering-gallery-mode (WGM) ${ }^{1}$ or morphology-dependent resonance (MDR) in the dielectric sphere with micrometer-sized droplets ${ }^{2,3}$ or solid particles. ${ }^{4.5}$ In addition, the high $Q$ value of spherical cavities has been shown to provide us with very lowthreshold lasers. ${ }^{2.3}$ This letter reports some features of WGM dye lasers from a single dye-doped solid sphere, operating in the blue, green, or orange spectral region, pumped by an ultraviolet (UV) $\mathbf{N}_{2}$ laser. To our knowledge, this is the first report on laser operation in blue and green spectra regions due to WGM using dye-doped solid spheres.

A schematic diagram of an experimental setup is shown in Fig. 1. A transversely excited atmospheric (TEA) UV $\mathrm{N}_{2}$ laser ( $337 \mathrm{~nm}$ ) is used to excite a spherical dye-doped WGM laser (DD-WGM), focusing the pumping UV $\mathrm{N}_{2}$ laser beam with a quartz spherical lens (QSL) after beam shaping through a circular beam aperture (CA).

Emission light from the DD-WGM laser is detected by a detector system (spectrum analyzer or biplanar phototube/oscilloscope system) via a quartz-glass optical fiber. A position of the DD-WGM laser, which is on a glass plate under a microscope, is adjusted finely in the vicinity of the focal point of the QSL with 5-cm focal length.

A dye-doped sphere is prepared by immersing a transparent polystyrene or an ion-exchange resin with a small diameter $(\sim 20-800 \mu \mathrm{m})$ in rhodamine 6G (RH-6G), fluorescein (sodium salt) (NA-F), or dimethyl-POPOP (DPOPOP) ethanol solution $\left(10^{-5}-10^{-3} \mathrm{~mol} / \mathrm{l}\right.$ ) for the orange, green, or blue spectral region, respectively, and by drying naturally in the room. Thus the single dye-doped sphere is excited by the UV $\mathrm{N}_{2}$ laser. It is recognized from the observation by a microscope that the dye-doped sphere has a good quality.

The laser-pulse wave forms of the TEA UV $\mathrm{N}_{2}$ laser and DD-WGM laser were measured with a calibrated $\mathrm{Ha}$ mamatsu Photonics R1193U-02 biplanar phototube and a Hewlett Packard 1744A storage oscilloscope (100 MHz) system. The spectral characteristics of the DD-WGM laser output pumped by the TEA UV $\mathrm{N}_{2}$ laser were analyzed with an optical multichannel analyzer [(OMA) Hamamatsu Photonics PMA-10].

The TEA UV $\mathrm{N}_{2}$ laser typically exhibits a 1.3-ns full width at half maximum (FWHM) and 3-kW peak power at $337 \mathrm{~nm}$ in the second positive band of $\mathrm{N}_{2}$ molecules, measured with a 275-MHz real-time oscilloscope and with a Photodyne 66XLA power/energy meter, respectively. The typical $\mathrm{N}_{2}$-laser pulse wave form is shown in Fig. 2, which is exhibited as a band-limited pulse by a $100-\mathrm{MHz}$ storage oscilloscope.

By finely adjusting the position of the dye-doped sphere in the vicinity of the focal point of the QSL, varying many bright-reddish full moon and crescent patterns in the near field were observed. In the far field, however, such patterns were never observed. This implies that the laser emission from the dye-doped sphere emerges in all radial directions in direct contrast to the unidirectional beam of low divergence produced by the usual dye lasers, ${ }^{3}$ and the DD-WGM laser serves as a coherent point source emitting in all directions in the far field. ${ }^{7}$

Figure 3 shows typically the laser and fluorescence wave forms from the RH-6G doped DD-WGM sphere, in the upper and lower traces, respectively, measured with a biplanar phototube and a $100-\mathrm{MHz}$ storage-oscilloscope system, corresponding to the higher and lower $\mathrm{N}_{2}$-laser pumping intensities induced by neutral density filters. The pulses between laser and fluorescence are clearly different, with respect to the rise of pulses. The laser pulse follows the pumping $\mathrm{N}_{2}$-laser pulse, while the fluorescent one exhibits the slower rise, which have a similar tendency in all dye/solvent systems.

In order to verify that the laser operation was due to WGM, we examined the mode structure of the emission

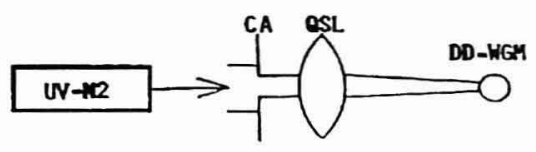

FIG. 1. Experimental setup of WGM dye laser system. UV-N2: TEA UV $\mathrm{N}_{2}$ laser; CA: circular beam aperture; QSL: quartz spherical lens; DDWGM: spherical dye-doped WGM laser. 


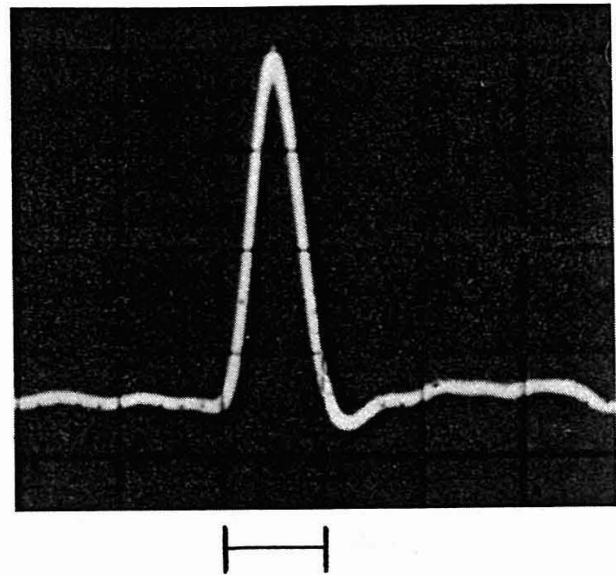

FIG. 2. Typical oscilloscope trace of UV $\mathrm{N}_{2}$-laser pulse measured with a $100-\mathrm{MHz}$ storage oscilloscope. The horizontal scale is $5 \mathrm{~ns} /$ div.

spectra from the dye-doped polystyrene sphere. Figure 4 shows the typical emission spectra, which are obtained above threshold from a single dye-doped sphere of RH-6G (orange), NA-F (green), or D-POPOP (blue region), with 65,33 , or $25 \mu \mathrm{m}$ diam, respectively. The lasing spectra exhibit a rather simple periodic structure, which may be restricted by a resolution $(\sim 1 \mathrm{~nm})$ of the OMA system.

The mode spacing of the observed spectra under the various diameters of the spherical DD-WGM laser is plotted in Fig. 5. The mode spacing is nearly proportional to the inverse diameter of the sphere. This indicates that the present laser operates according to the WGM in the spherical cavity. The solid straight line is obtained when the refractive index is $n=1.6$ for the polystyrene spheres. We have also observed that the rim of the dye-doped sphere above threshold is brighter than the center, while the image of the emission pattern is rather uniform below threshold.

In conclusion, we have examined the emission from dye-doped small spheres pumped by TEA UV $\mathrm{N}_{2}$ laser. Temporal, spectral characteristics and spatial images of the emission clearly show the features of the WGM laser with

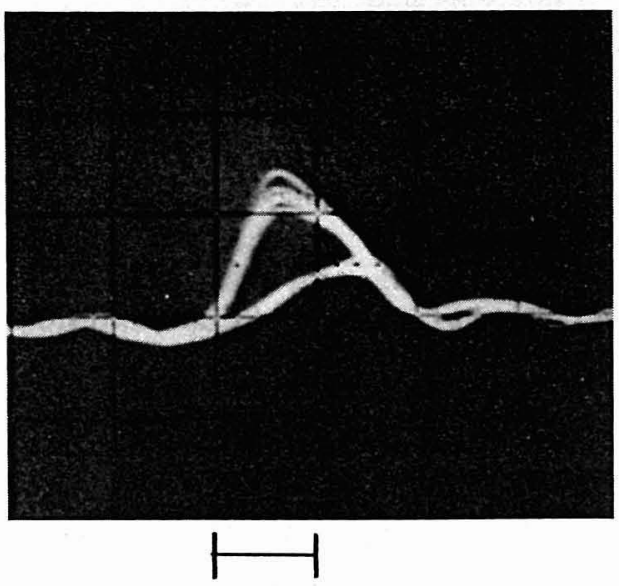

FIG. 3. Laser and fluorescence wave forms from RH-6G doped DDWGM in the upper and lower traces, respectively. The horizontal scale is 5 ns/div.
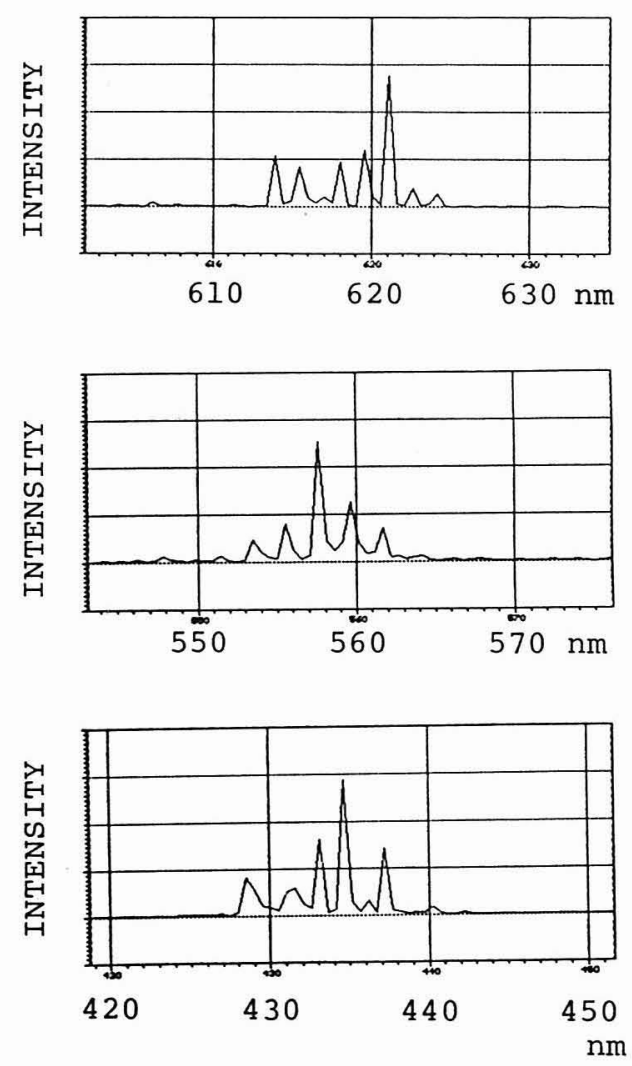

FIG. 4. Typical WGM spectra obtained from the spheres of the RH-6G (orange), NA-F (green), and D-POPOP (blue regions), with 65, 33, and $25 \mu \mathrm{m}$ diam from top to bottom, respectively.

high $Q$ values. The solid spherical WGM laser output in the green and blue spectral regions, from the NA-F and D-POPOP dyes, respectively, is the first report. The technique of the dye-doped spherical WGM dye laser reported here may be applied to obtain various spectra and theory/ experimental comparisons in the WGM laser using dielectric small spheres.

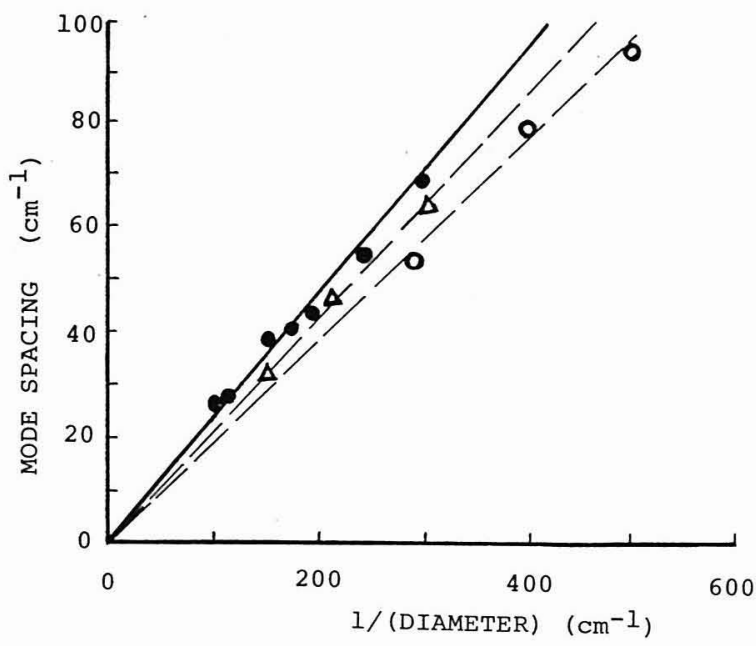

FIG. 5. Mode spacing of measured spectra under the various diameters of the DD-WGM laser. Solid circles: RH-6G; triangles: NA-F; open circles: D-POPOP. The solid straight line is obtained when the refractive index is $n=1.6$ for the polystyrene spheres. 
The authors would like to express their thanks to Professor H. Inaba of Tohoku Technical University for his helpful suggestions. This work was partly supported by the 1991 Grant-in-Aid of the Special Project for Educational Research, Iwate University.

${ }^{1}$ C. G. Garrett, W. Kaiser, and W. L. Bond, Phys. Rev. 124, 1807 (1961).

${ }^{2}$ H.-M. Tzeng, K. F. Wall, M. B. Long, and R. K. Chang, Opt. Lett. 9, 499 (1984).
${ }^{3}$ H.-B. Lin, A. L. Huston, B. L. Justus, and A. J. Campillo, Opt. Lett. 11, 614 (1986).

${ }^{4}$ R. E. Benner, P. W. Barber, J. F. Owen, and R. K. Chang, Phys. Rev. Lett. 44, 475 (1980).

${ }^{5}$ M. K. Gonokami, K. Takeda, H. Yasuda, and K. Ema, Jpn. J. Appl. Phys. 31, L99 (1992).

${ }^{6}$ H. Taniguchi, H. Yamada, T. Fujiwara,S. Tanosaki, H. Ito, H. Morozumi, and M. Baba, Jpn. J. Appl. Phys. 32, L58 (1993).

${ }^{7}$ S.-X. Qian, J. B. Snow, H.-M. Tzeng, and R. K. Chang, Science 231, 486 (1986). 